\author{
Giuliana Antonella Pignataro ${ }^{1}$ \\ 0000-0001-5129-6620 \\ Laboratorio de Investigación en Ciencias \\ Humanas. \\ Universidad Nacional de San Martín, \\ Argentina.
}

\section{Alumnas-madres. \\ Experiencias de maternidad y escolaridad en la escuela secundaria}

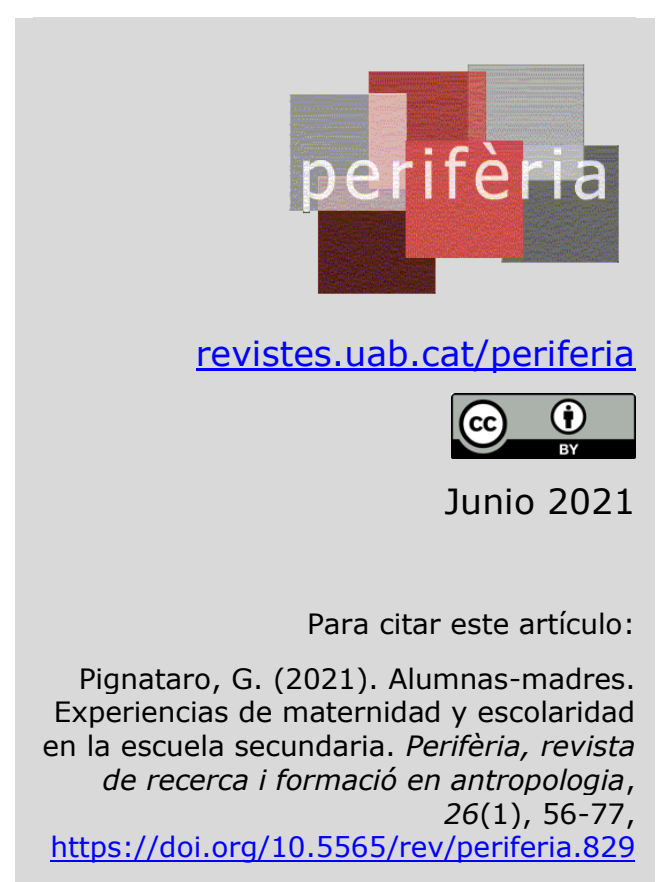

Pignataro, G. (2021). Alumnas-madres. Experiencias de maternidad y escolaridad la escuela secundaria. Perifèria, revista 26(1), 56-77, https://doi.org/10.5565/rev/periferia.829

\title{
Resumen
}

El objetivo de este trabajo consiste en analizar, desde una perspectiva antropológica, los modos en que las experiencias de maternidad y maternaje de un grupo de adolescentes escolarizadas de un barrio popular de Buenos Aires entran en tensión con las miradas del cuerpo docente en relación a la maternidad en la adolescencia como algo "problemático". Además, se indaga en el rol que ocupa la institución escolar en dichas experiencias de maternidad. Para ello, se asistió de manera periódica a la escuela, participando en clases de diversas asignaturas, así como también se realizaron entrevistas informales y semiestructuradas a docentes de la escuela, y a estudiantes. Del análisis surge un choque de perspectivas. Esto es, la escuela se configura como un espacio ambiguo que intenta acompañar las experiencias de maternidad al mismo tiempo en que entra en tensión con ellas. Las jóvenes, por otra parte, desafían estas miradas, reivindicando su derecho a ser madres y desplegando modos particulares de habitar la escuela siendo madres adolescentes.

Palabras clave: Maternidades populares; Escuela; Adolescencia; Interseccionalismo; Antropología social.

\footnotetext{
${ }^{1}$ Contacto: Giuliana Antonella Pignataro - giulianapignataroar@gmail.com
} 
Abstract: Student-Mothers. Maternity and schooling experiences in a secondary school

The objective of this work is to analyze, from an anthropological perspective, the ways in which the maternity and maternal experiences of a group of schooled adolescents in a popular neighborhood in Buenos Aires come into tension with the views of the teaching staff in relation to the motherhood in adolescence as something "problematic". In addition, the role of the school institution in these maternity experiences is investigated. To do this, the field work consisted in attending the school regularly, participating in classes of various subjects, and analyzing informal and semi-structured interviews with school teachers and students as well. From the analysis emerges a clash of perspectives. That is, the school is configured as an ambiguous space that tries to accompany the experiences of motherhood at the same time that it enters into tension with them. Young women, on the other hand, challenge these views, claiming their right to be mothers and displaying particular ways of living in the school as teenage mothers.

Keywords: Popular maternities; School; Adolescence; Interseccionalism; Social Anthropology.

\section{Introducción}

En este texto se analizan los modos en que las experiencias de maternidad y maternaje de un grupo de adolescentes, de entre 14 y 19 años de edad, entran en tensión con las miradas de les adultes ${ }^{2}$ al interior de la escuela. El objetivo consiste en comprender las distintas percepciones que el cuerpo docente posee en relación a la maternidad en la adolescencia y determinar en qué medida esas maternidades constituyen (o no) un problema desde la mirada institucional.

Los datos que aquí se presentan forman parte de una investigación cualitativa cuyo resultado fue una tesina de grado. Su objetivo consistió en analizar las experiencias

\footnotetext{
${ }^{2}$ A lo largo del texto se utilizará lenguaje inclusivo a la hora de hacer referencia a distintos grupos de personas. La utilización del morfema -e constituye una variante (más amigable en la pronunciación) del uso de la "x" o la "@". La finalidad del uso de este tipo de lenguaje consiste en romper no sólo con el sexismo que acarrea el génerico masculino sino también con los binarismos femenino/masculino.
} 
de feminidad relacionadas al trabajo materno y de cuidados de un grupo de adolescentes escolarizadas de un barrio popular de Buenos Aires, prestando particular atención a los modos en que dichas prácticas eran percibidas por las jóvenes como espacios de agencia, goce y satisfacción personal. Parte de los datos recabados en dicho trabajo se presentan en este artículo cuyo objetivo consiste en analizar los modos en que las experiencias de maternidad y maternaje de las adolescentes entran en tensión, en muchos casos, con las miradas del cuerpo docente.

Los resultados que se presentan en este texto se basaron en el análisis de datos producidos durante un trabajo de campo en una escuela pública que se extendió durante un año y medio, y que implicó mi asistencia semanal a la institución. Allí se realizaron observaciones en clases de diversas asignaturas, así como también entrevistas informales y semiestructuradas a docentes y estudiantes de la escuela. La escuela se encuentra ubicada en un distrito del Área Metropolitana de Buenos Aires (AMBA) ${ }^{3}$. El barrio donde se encuentra emplazada la escuela se caracteriza por una situación de pobreza urbana, densidad poblacional y degradación ambiental. La escuela es la única escuela pública del barrio y allí asisten tanto estudiantes vecines del propio barrio como de barrios lindantes. Mi llegada a la institución se enmarca en mi inserción en un espacio de investigación interdisciplinario, el CEDESI (Centro de Estudio sobre Desigualdades, Sujetos e Instituciones) perteneciente a la Universidad de San Martín, el cual posee una trayectoria de más de 10 años de trabajo tanto en el barrio como en la escuela. Dicha presencia semanal me permitió entablar relaciones cercanas con las estudiantes, así como también conocer las dinámicas de la escuela y los sentidos que circulaban por ella. A su vez, la realización de entrevistas a algunas estudiantes me permitió acceder a un espacio más íntimo en donde las chicas pudieran charlar sobre sus experiencias de maternidad. Las estudiantes entrevistadas fueron aquellas con las que entablé una relación más cercana. Las entrevistas fueron individuales y las chicas fueron informadas sobre la investigación y la temática de estudio, manifestando un consentimiento oral en donde se garantizó el anonimato tanto de sus nombres, el nombre del barrio y de la escuela.

\footnotetext{
${ }^{3}$ El AMBA es la zona urbana común que conforman la Ciudad de Buenos Aires y los siguientes 40 municipios de la Provincia de Buenos Aires.
} 
Las preguntas a las que procuro dar respuesta en este artículo son: ¿Qué percepciones posee el cuerpo docente en relación a la maternidad en la adolescencia? ¿Cuál es el rol de la escuela en estas experiencias escolares y de maternidad? La idea que sostengo es que la escuela se configura como un espacio ambiguo que intenta acompañar las experiencias de maternidad de las jóvenes al mismo tiempo en que entra en tensión con ellas. Las adolescentes, por otra parte, desafían estas miradas, reivindicando su derecho a ser madres y desplegando modos particulares de habitar la escuela siendo madres y adolescentes.

En una primera parte del texto, se discuten las propuestas teóricas desde las que se ha abordado la maternidad en la adolescencia y, más específicamente, en los sectores populares. En una segunda parte, se analizan las distintas perspectivas que posee el cuerpo docente en relación a las experiencias de maternidad de las estudiantes, indagando en qué medida estas experiencias constituyen un "problema a solucionar".

\section{Abordajes teóricos de la maternidad en la adolescencia}

La maternidad y el embarazo en la adolescencia son temáticas que vienen siendo estudiadas desde diversas disciplinas ${ }^{4}$ y perspectivas desde aproximadamente cinco décadas $^{5}$. Estos trabajos pueden ser clasificados, de una manera general, en tres grandes grupos. Por un lado, en aquellos trabajos tradicionales que consideran la maternidad en la adolescencia como situaciones que acarrean siempre consecuencias negativas para las adolescentes, tales como el abandono de los estudios, la perpetuación de la pobreza, la exclusión social, la reclusión en el hogar, e incluso el consumo de alcohol y drogas (Rondón y Betancur, 2015; Posada, 2014; Espinosa, 2020). En un segundo grupo, podemos ubicar aquellos trabajos que, desde una perspectiva revisionista (en la cual se enmarca este trabajo), intentan distanciarse

\footnotetext{
${ }^{4}$ La demografía (Unicef, 2019; Pantelides y Binstock, 2007; Cono sur, 2017), la medicina (Langer, 2002; Posada, 2014; Barrett y Wellings, 2002), la psicología social (Czerniuk, 2016), la sociología (Betancur y Rondón, 2015, Zamberlín, 2005), y antropología (Adaszko, 2005; Alvarez y Perez, 2015) entre otras, son algunas de las disciplinas que han estudiado la maternidad y paternidad en la adolescencia.

${ }^{5}$ La tematización del embarazo adolescente como un "problema" de salud pública, merecedor de atención en términos investigativos, surge en la década del 60 acompañando los cambios sociodemográficos, económicos, culturales e ideológico-políticos que tuvieron lugar tras la Segunda Guerra Mundial en Estados Unidos. Para acceder a más información sobre la temática, ver Furstenber, 2003; y Geronimus, 2003.
} 
de las miradas adultocéntricas y de los prejuicios en torno al embarazo en la adolescencia que analizan las prácticas culturales de les jóvenes desde su propia perspectiva (Adaszko, 2005), y proponen reflexionar acerca de cómo se interpreta y aborda dicho fenómeno desde las instituciones (Casas Alvarez y Solorio Perez, 2015; Adaszko, 2005; Czerniuk, 2016). Finalmente, entre ambas perspectivas, encontramos aquellos trabajos que, si bien intentan desligarse de estas miradas adultocéntricas, haciendo hincapié en las perspectivas de las adolescentes y sus deseos, aún continúan calificando el embarazo en la adolescencia como "precoz" o "temprano" (Parra Abaúnza, 2012; Mariño, 2013; Weisbrot et. al., 2019) y señalando a las adolescentes madres como una "población vulnerable de alto riesgo social" (Mariño, 2013). Desde estos abordajes, si bien se considera que algunos embarazos en la adolescencia pueden ser "deseados", dicho deseo se minimiza aduciéndolo a la pobreza y la falta de oportunidades, y se propone que los Estados y las instituciones posean una injerencia activa a la hora de evitar que las adolescentes se embaracen.

Sin embargo, la maternidad durante la adolescencia no siempre se consideró problemática. Durante varias décadas y en diversos contextos culturales tener hijes a edades "tempranas" constituyó un esquema normativo de reproducción ${ }^{6}$. Diversas transformaciones socioculturales recientes, tales como la masificación de la educación, el cuestionamiento de los roles de género, la creciente participación de las mujeres en el ámbito público, y la conceptualización de la adolescencia como un periodo problemático de transición hacia la adultez, han contribuido a visualizar la maternidad en la adolescencia como un problema el cual debe ser resuelto a través de la injerencia de las instituciones (Llanes Díaz, 2012).

Adaszko (2005) cuestiona la categoría de problema relacionada con el embarazo en la adolescencia y sostiene que el "problema embarazo adolescente" se encuentra fuertemente atado a la o las formas en que pensamos la adolescencia y la juventud. Al mismo tiempo, señala que es el campo científico y político quienes estigmatizan el

\footnotetext{
${ }^{6}$ Por lo menos en Latinoamérica, hasta fines del siglo XIX era común el casamiento a "temprana" edad y, por consiguiente, la maternidad en el periodo que hoy conocemos como adolescencia. Esta transformación se encuentra íntimamente ligada con el desarrollo del concepto moderno de "adolescente" surgido en la primera mitad del siglo XIX en las sociedades occidentales. Dicha transformación se produjo de forma gradual a partir de la Revolución Industrial debido a la universalización de la escolarización de la población y a la correspondiente delimitación de edad para pasar por la escuela. Para más información sobre el surgimiento del concepto de adolescencia, ver Stern y García, 2001.
} 
embarazo en la adolescencia, estableciendo modos de controlar la sexualidad de les adolescentes, especialmente de las mujeres pobres, y obviando ciertos aspectos positivos de estas experiencias. Desde el presente trabajo, entonces, se plantea que es urgente analizar aquellos aspectos no tan difundidos de la maternidad en la adolescencia, tales como su papel en ciertas construcciones identitarias, la motivación por estudiar y/o encontrar un empleo, así como el reconocimiento y el prestigio social que puede implicar en el entorno. A su vez, ni la edad, ni la maternidad, son factores que determinan condiciones de pobreza. Por el contrario, son factores estructurales, económicos y sociales de la sociedad que habitamos los que generan condiciones factibles para la perpetuación de las desigualdades sociales $y$, por consiguiente, de la desigualdad de oportunidades que sufren muchas jóvenes adolescentes (Nóblega, 2009; Oviedo \& García, 2011; Pantelides, 2004).

De esta manera, teniendo en cuenta estas discusiones teórico conceptuales, en el presente trabajo me propuse analizarlos modos en que las experiencias de las estudiantes entran en tensión con las perspectivas del cuerpo docente en relación al embarazo en la adolescencia. El argumento central que será abordado a lo largo del texto consiste en que la escuela se configura como un espacio ambiguo que intenta acompañar las experiencias de maternidad de las estudiantes al mismo tiempo en que entra en tensión con ellas.

\section{Controles, chistes y retos}

Durante mis primeras visitas a la escuela noté que algunas adolescentes que asistían quedaban embarazadas. En una primera instancia, desde mi perspectiva, de persona adulta y de clase media, consideré que dichos embarazos, para mí "tempranos" y "no buscados", implicaban un impedimento para el desarrollo personal y académico de las jóvenes y, por lo tanto, representaban algo negativo. Sin embargo, a medida que fui complejizando mi mirada e interactuando más cercanamente con las estudiantes, comencé a observar que las chicas vivían esa maternidad con alegría y, además, si bien la mayoría de esos embarazos no habían sido buscados, eso no necesariamente implicaba que no fueran deseados. Ante esto, comencé a preguntarme por qué consideraba estos embarazos como problemáticos cuando las chicas mismas no lo veían de esa manera. Las jóvenes con las que interactué durante el trabajo de campo 
suelen ser hijas de mujeres que fueron madres en la adolescencia o en los primeros años de la adultez. También es importante señalar que las estudiantes suelen realizar tareas de cuidado y maternaje desde muy temprana edad, cuidando de sus hermanites, primites más pequeñes, o contrayendo trabajos remunerados cuidando de les hijes de vecines del barrio.

Si bien no todes les docentes comparten la misma mirada en relación a los embarazos en la adolescencia, para algunes de elles, la maternidad en la adolescencia es fruto de un desconocimiento de la salud sexual y reproductiva y, sobre todo, del (mal) uso de métodos anticonceptivos. Los embarazos son considerados no deseados, accidentales y producto de una falta de información sobre cómo cuidarse. Las charlas entre estudiantes y el cuerpo docente sobre maternidades en la adolescencia suelen evidenciar cierta disputa por calificar esas experiencias como deseadas/no deseadas. Una mañana en la escuela, presencié una conversación de este tipo en donde una docente, Amelia", daba un consejo sobre cuidarse para "evitar maternidades y paternidades no deseadas" y una estudiante, Lourdes, le respondía interpelándola de manera directa sobre qué haría si ella, la docente, quedara embarazada sin planearlo.

Amelia: Por eso yo les digo "cuídense". Después tienen hijos que no desean (...)

Tienen que aprovechar cuando son jóvenes para estudiar, viajar. Si tenés un

hijo no se puede.

Lourdes: ¿Vos tenés hijos? (A Amelia)

Amelia: Nooo y no quiero tener.

Lourdes: Y si quedás embarazada, ¿qué hacés?

Amelia: Naaa, si te cuidás con pastillas y con preservativo no quedás.

Lourdes: Buen, pero ¿y si quedaras?

Amelia: No quedás, chicos. Eso no pasa.

En esta conversación con Amelia, Lourdes insistía para saber si la profesora consideraría abortar en el caso de quedar embarazada sin planearlo, y la docente

\footnotetext{
7 Todos los nombres de las personas mencionadas han sido modificados con la finalidad de preservar la identidad de quienes colaboraron con esta investigación.
} 
intentaba evitar dar esa respuesta ya que el aborto, suele ser una práctica muy condenada por las adolescentes con las que me encontré en el campo. De alguna manera, indica una maternidad no deseada, lo cual es visto como algo extraño por las estudiantes. Incluso, aquellos embarazos no planeados, los que son calificados por la docente como "hijos que no desean", son caracterizados como "deseados" por las chicas.

Esta visión de parte del cuerpo docente - del embarazo en la adolescencia como "no deseado", siempre accidental y problemático- deriva en una serie de prácticas y discursos, que se manifiestan en controles y retos, cuyo fin último es evitar que las maternidades en la adolescencia sucedan. Estos controles de parte de las docentes distan de ser violentos o de intentar incomodar o avergonzar a las estudiantes. Por el contrario, se constituyen como prácticas de cuidado cuyo fin último es lograr que las alumnas terminen sus estudios secundarios y, hasta quizás, continúen con una profesionalización terciaria o universitaria. En Argentina, la obligatoriedad de los estudios secundarios se establece en el año 2006 con la Ley Nacional de Educación 26.206. Dicha ley fue sancionada con la finalidad de consolidar la idea de la educación secundaria como un derecho. De alguna manera, este despliegue de discursos y prácticas de les docentes responden a un cuidado que se relaciona con la necesidad de garantizar el derecho educativo de las estudiantes, así como también con la esperanza depositada en la profesionalización como vía de ascenso social o de mejoras en las condiciones de vida.

Así, en la cotidianeidad de la escuela suelen producirse intercambios entre docentes y estudiantes donde, muchas veces, el disparador suele ser la broma de una alumna sobre un embarazo o maternidad inexistente. Las respuestas de las docentes suelen ser retos y preguntas en relación al uso de anticonceptivos. Tal como sostiene Fainsod (2008), la literatura académica tradicionalista nos ha dejado una visión sobre el embarazo en la adolescencia que lo vincula intrínsecamente con el abandono de proyectos "típicamente juveniles" como el educativo ${ }^{8}$. Estas visiones refuerzan un

\footnotetext{
${ }^{8}$ Algunos estudios revisionistas (Climent, 2003; Llanes Díaz, 2012) cuestionan la relación automática entre embarazo en la adolescencia y el abandono de los estudios secundarios, señalando que muchas veces la deserción escolar sucede antes del embarazo. A lo largo de mi trabajo de campo, presencié varios casos donde las jóvenes quedaban embarazadas no estando escolarizadas, así como también algunas de ellas retomaban sus estudios luego de ser madres.
} 
"deber ser" adolescente en donde la maternidad y paternidad actúan como "desvíos". Es decir, los embarazos se conciben como fenómenos que no deberían ocurrir en ese momento vital. En este marco, los retos de las docentes podrían leerse sólo como disciplinadores, pero aquí propongo pensarlos, también, como prácticas de cuidado cuyo fin último es lograr que les adolescentes terminen sus estudios secundarios.

Al mismo tiempo, el hacer un chiste sobre una maternidad inexistente es una forma de desafiar estas miradas institucionales que establecen una relación negativa entre maternidad y adolescencia. Ser adolescente es incompatible con ser madre ya que, en parte, las tareas de la maternidad "dificultan" las actividades escolares. De la siguiente manera lo ilustraba Ricardo, el director de la institución, quien previamente se encontraba charlando conmigo sobre la experiencia de maternidad de Sabrina, una estudiante que había sido madre hacía unos meses y asistía a la escuela sólo una o dos veces al mes. En el siguiente extracto de entrevista, vemos cómo Ricardo contrapone el caso de Sabrina con el de Carla, una alumna-madre que continuó sus estudios sin muchas dificultades y logró mantenerse en el rol de "sólo adolescente" que la escuela esperaba de ella.

Ricardo: "Pero sí destaco que, te decía, Carla... que ella sí pudo hacerlo. Tuvo la madre que la mandó. $Y$ ella acá era una adolescente más. Y no quería interponer que era mamá con el resto, porque fueron 9 meses. La única diferencia que tuvo con el embarazo. Entonces acá venía a ser adolescente y a hacer lo que tenía que hacer".

Al asistir a clases como cualquier otra estudiante, Carla estaba siendo "una adolescente más" e iba a la escuela a "hacer lo que tenía que hacer". En este sentido, la figura de alumna-madre rompe con la de adolescente que la escuela espera de sus alumnas. Sin embargo, las chicas desafían dicho deber ser de diversas maneras: algunas estudiantes madres, como Sabrina, priorizan el trabajo materno y de cuidados, asistiendo de manera intermitente a la escuela; otras, lo hacen a través de la palabra, en los intercambios cotidianos de la escuela. La comunicación y el desafío a través del humor, como mencioné anteriormente, es algo que noté que sucedía con gran frecuencia en la escuela. Sobre todo, las chicas, que es con quienes más interactuaba, solían hacer chistes con frecuencia sobre estar embarazadas. Lo mencionaban entre risas para, luego, ante mi sorpresa, desmentirlo: "Ay, profe. Es un chiste". De la siguiente manera, Ricardo, el director de la escuela, me comentaba 
una de estas situaciones:

Ricardo: "De hecho, hay una nena que está... hace dos o tres días que está diciendo que está embarazada, que está embarazada... Pero está diciendo que está embarazada en chiste. Viste cuando hablás del tema... pero hay algo ahí (...) hace tres días, estamos comiendo, y juega, juega, y juega. 'Yo ya soy grande, yo ya sé lo que tengo que hacer'".

Para Gómez Capuz (2002 en Ojeda Álvarez y Cruz Moya, 2004), el humor se define como la transgresión consciente, deliberada y sistemática de los mecanismos que rigen el normal desarrollo de la interacción comunicativa cotidiana. Esto quiere decir que implica la superación de una serie de expectativas que les hablantes comparten a la hora de establecer una comunicación. El chiste es un elemento dinámico con una enorme potencialidad expresiva que sirve para mantener la atención de le oyente, por lo que también tiene el efecto de entablar y mantener relaciones sociales (Guerin, 2003 en Ojeda Álvarez y Cruz Moya, 2004). No obstante, las relaciones de comunicación no sólo constituyen intercambios lingüísticos sino relaciones de poder simbólico en donde tienen lugar actualizaciones de las relaciones de fuerza entre les interlocutores (Bourdieu, [1985]2001). El hecho de comunicar, a través del humor, el deseo de ser madres intenta romper con la posición de sólo adolescentes (es decir, que se dedican exclusivamente a ser estudiantes y no a maternar) que la escuela asigna e intenta instalar en las estudiantes. De alguna manera, es a través de los chistes que las estudiantes se auto-reposicionan, en el acto del habla, en la figura de alumnas-madres, al mismo tiempo que valorizan dicha posición. La estudiante que dice estar embarazada lo hace acompañada de una frase que indica que el trabajo materno es valorado de manera positiva: "ya soy grande, yo ya sé lo que tengo que hacer". En ese sentido, ser madre implica una gran responsabilidad y, por lo tanto, un crecimiento hacia la adultez (Nóblega, 2009).

Así, como les docentes despliegan esta serie de retos hacia aquellas estudiantes que se salen de la norma de ser sólo adolescentes, también dedican esfuerzos en evitar dichos embarazos interviniendo en las relaciones afectivas que se dan dentro del 
espacio escolar ${ }^{9}$ a través de una serie de controles. Las intervenciones consisten en consejos que indican con quién conviene mantener una relación sexo-afectiva y de qué manera. Así, Soledad, una estudiante de $4^{\circ}$ año me contaba sobre un chico que asistía a la escuela y que le gustaba.

Soledad: "Me da cosa venir acá10 porque hay muchos pibes que sé que gustan de mí"

Giuliana: "¿Y vos qué onda?"

Soledad: "No, yo nada que ver (...) Ya se tienen que dar cuenta con mi cara. Yo no les doy pelota. Aparte a mí me gusta otro"

Giuliana: "¿Quién te gusta?"

Soledad: "Fabrizio, el chiquitito"

Giuliana: "¿Y qué onda? ¿Pasó algo?"

Soledad: "Sí, pasó, pasó" (se sonríe). "Pasa que nosotros no queremos ser novios porque nos llevamos bien y tenemos miedo que después termine todo mal. Así que somos amigos con derechos" (se ríe).

Giuliana: "Bueno... está bien eso"

Soledad: "Ramona [docente de la escuela] nos dice que no. Que nos tenemos que poner de novios"

Giuliana: "¿Por qué?"

Soledad: "Porque piensa que nos vamos a llenar de hijos" (se ríe)

Ese mismo día, en mi presencia, Ramona le dio el visto bueno a Soledad sobre Fabrizio, le dijo que era un "pibe bueno" y que le haría bien estar con él. En el relato de Soledad podemos ver que, si bien Ramona aprueba esa relación, no aprueba su forma. Para ella las relaciones sexuales sin un compromiso pueden llevar a maternidades adolescentes no deseadas. Como podemos observar aquí, la escuela

\footnotetext{
${ }^{9}$ Es importante destacar que estos esfuerzos no son muy efectivos ya que la gran mayoría de las relaciones sexo-afectivas que derivaron en embarazos durante mi trabajo de campo se dieron con varones que asistían a otras escuelas o que no estaban escolarizados.

${ }^{10}$ Soledad se refiere al espacio deportivo que funcionaba en la escuela los días sábados por la mañana.
} 
adopta la función, por un lado, de prevenir esos embarazos y también, como veremos más adelante, de re-encauzarlos (Fainsod, 2008). Sin embargo, tanto las prácticas que poseen la finalidad de prevenir (entrega de preservativos, clases de educación sexual, intervención en relaciones afectivas) como aquellas que buscan re-encauzar (contener a las jóvenes dentro de las aulas, incentivarlas a continuar estudiando) no se configuran solamente como dispositivos de control y disciplinamiento, sino también como prácticas de cuidado. Esto resulta indispensable analizarlo teniendo en cuenta las lógicas gerenciales que rigen la vida en los barrios populares, donde tanto instituciones como sujetes deben desarrollar estrategias de supervivencia de manera cotidiana (Grinberg \& Dafunchio, 2013). La gestión del sí en estas condiciones y la esperanza depositada en la educación como forma de acceso a ciertas posibilidades de ascenso social, derivan en docentes e instituciones educativas intentando evitar, contra viento y marea, el abandono escolar.

En este sentido, planteo la escuela no sólo como un panóptico (Muñoz, 2017; Scharagrodsky, 2005) sino como un espacio donde se individualiza el cuidado hacia les sujetes. Diversos elementos que a simple vista identificaríamos como disciplinadores se configuran como prácticas de cuidado que se sostienen sobre una visión adultocentrista en torno al deber ser adolescente pero que poseen como fin último evitar que las cosas se "salgan de control" (Grinberg, 2009). Es a través de los chistes y los comentarios en tono lúdico en el ámbito escolar que las chicas desafían esas perspectivas, corriéndose del lugar de "adolescentes" que la escuela les asigna para reposicionarse en el de "alumnas-madres" en donde se ensamblan diversos elementos identitarios tales como estudiante secundaria, adolescente y madre.

\section{El embarazo en la adolescencia como "problema"}

Como mencioné anteriormente, la maternidad en la adolescencia ha sido una temática ampliamente estudiada desde la academia y muchos de sus abordajes han llevado a que se la conceptualice como problemática. La mayoría de las investigaciones se preguntan por los factores que contribuyen a que les adolescentes inicien relaciones sexuales, por los elementos que inciden en el uso inconsistente de métodos anticonceptivos y por las causas y consecuencias del embarazo, la 
maternidad y la paternidad en la adolescencia. Los enfoques más tradicionales, desde la salud, sostienen que les jóvenes no poseen la suficiente madurez psicológica ni las capacidades necesarias, que sí poseerían les adultes, para evaluar las consecuencias de sus actos. Al mismo tiempo, arriban a la conclusión de que el embarazo en la adolescencia acarrea consecuencias negativas sobre la salud y el bienestar de les niñes ya que les jóvenes serían incapaces de asumir adecuadamente su cuidado y crianza. En esta misma línea, se sostiene que la maternidad en la adolescencia implicaría que las jóvenes vean coartadas sus posibilidades de desarrollo personal lo cual, inevitablemente, las condenaría a permanecer o caer en la pobreza (Pantelides, 2004, García Hernández, 2014; Vélez \& Figueredo, 2015). Otres autores sostienen que la pobreza y la falta de oportunidades educativas y laborales serían la causa y no la consecuencia de la maternidad en la adolescencia (Stern y García, 2001; Oviedo y García, 2011). En conclusión, desde estas perspectivas, se entiende el embarazo en la adolescencia como un problema de carácter público en donde la opinión de les adultes tiene mayor peso que la de les jóvenes. En este sentido, una de las inquietudes que busco responder es cuáles son las distintas percepciones que el cuerpo docente posee sobre la maternidad en la adolescencia y en qué medida estas maternidades consideradas tempranas constituyen (o no) un problema desde la mirada institucional.

Durante una entrevista realizada a Ricardo, el director de la escuela, le pregunté si consideraba que la maternidad en la adolescencia representaba un problema. Su primera respuesta fue la siguiente:

Ricardo: "Yo supongo que no es un problema fundamentalmente por lo que veo. Porque en mi escuela (...) nosotros trabajamos con el $99 \%$ de los chicos de acá [del barrio], entonces tenemos un montón de alternativas que les podemos ofrecer para que no interrumpan las clases y todas esas cosas. Así que problema no es, y como escuela la verdad es que los tenemos que apoyar, no podemos decirles que no. Pero nosotros tenemos un formato que se va adaptando a las circunstancias que se necesiten, entonces te diría que no. Ahora en otra escuela que quizás son muchos más, que vienen de diferentes lados, tal vez pueda ser un problema porque no lo podés abordar desde la escuela tan fácil. Acá al ser menos nos conocemos todos (...) Entonces por eso te diría que no. Tal vez otras escuelas, sí". 
Aquí Ricardo diferencia a su escuela de otras. Él sostiene que, debido a la cantidad de alumnes (que es poca en comparación a otras escuelas) y al hecho que prácticamente todes les estudiantes son vecines del barrio, hay una mayor cercanía con el estudiantado. Ante cualquier problema les docentes se pueden acercar al domicilio de la alumna embarazada, charlar con sus familiares, y llegar a un acuerdo para que la estudiante no deje los estudios. A su vez, sostiene que el rol de la escuela es apoyar a las alumnas. Esto implica que la escuela se adapte a las circunstancias y a la situación que la joven en cuestión esté atravesando con la finalidad de que no deje de asistir a clases. Ricardo hace hincapié en la cercanía que la escuela tiene con sus alumnas y la capacidad que ésta posee de ofrecer opciones para que las chicas no abandonen la escuela. Sostiene que, en otras instituciones, donde asisten jóvenes de diferentes barrios y que cuenta con una mayor cantidad de matrículas, estas situaciones pueden representar un problema. Pero no es el caso de su escuela.

Ricardo: "Tenemos un plan para madres, para amamantamiento, ¿me entendés? Que las chicas pueden ingresar más tarde o irse antes, son normativas que existen ${ }^{11}$. Ellas para la asistencia tienen que venir y se pueden ir antes en el tiempo de lactancia, antes o después según como se organicen. O pueden entrar en el recreo, el bebé, la abuela [del bebé], para que amamante un rato acá, de ser necesario (...). Por eso desde la escuela te diría que no es un problema."

Hasta aquí pareciera que las jóvenes continúan sus estudios con total normalidad sin ningún tipo de problema. Ricardo comenta cuáles son las opciones que la escuela ofrece a las mamás para que no interrumpan sus estudios durante el periodo de amamantamiento. El director enfatiza que el embarazo en la adolescencia no representa un problema para la institución en la medida en que ésta ofrece toda una serie de opciones para que las chicas continúen asistiendo y, además, en tanto posee características particulares que la acercan a las estudiantes (su poca cantidad de matrículas y su alumnado proveniente del mismo barrio donde se encuentra

\footnotetext{
${ }^{11}$ En la Ciudad de Buenos Aires, las madres -y padres- adolescentes en edad escolar cuentan con derechos especiales, como un régimen más flexible de inasistencias (Ley $n^{\circ} 25.273$ ) y de prioridad para las vacantes en jardines maternales, que intentan garantizar la continuidad de estas jóvenes en el sistema educativo (Ley $n^{\circ}$ 709). En la provincia de Buenos Aires, la ley 14.637 también establece un régimen especial de inasistencias justificadas para alumnas embarazadas y alumnos en condición de paternidad que cursen en establecimientos de gestión estatal o privados.
} 
emplazada). Sin embargo, a medida que continuamos charlando y comentando casos particulares de estudiantes que fueron mamás, comienza a delinearse "el problema".

Ricardo: "Pero sí me di cuenta que les cuesta, necesitan dedicarse. Mirá que tengo casos de buenas alumnas. Por ejemplo, este año tienen que estar acá y no vienen. Porque fueron mamás a principio de año y sí. Me pasó... dos hay. Una que tuvo un embarazo complicado. Que después estuvo internada, entonces bueno, se recuperó, vino, saludamos, conocimos [al bebé], todo, y bueno, ahí se dio el problema, porque el problema es que yo me tengo que asegurar de que esté en la escuela. Y se lo aseguro, viste, agarradito con un hilo, con trabajos y esas cosas, pero... y es una buena alumna, una chica que yo no me hubiera imaginado que de pronto vaya a tomar la decisión de nada, pero hoy por hoy es nada ${ }^{12}$. Entonces es una chica que está en riesgo pedagógico porque no sé si va a pasar de año, si no se pone las pilas, aunque sea este último tiempo."

Aquí, Ricardo comienza a delinear la diferencia entre el embarazo como problema desde la institución y el embarazo como problema desde las alumnas-madres. El problema, según el director, no existe desde la escuela, la cual brinda las posibilidades a su alcance para que las jóvenes continúen sus estudios, sino desde las estudiantes que no asisten a clases luego de dar a luz. Sin embargo, el director no se desliga de dicha situación, sino que hace lo posible para poder asegurar esa continuidad: "se lo aseguro con trabajos y esas cosas (...) agarradito con un hilo". Resulta interesante pensar en el rol que desempeña la escuela en las experiencias de maternidad de las adolescentes: si bien existe un seguimiento pedagógico cercano e individualizado que resulta vital para la continuidad de los estudios de las chicas, la visión institucional sobre estas maternidades como problema entra en tensión con las perspectivas y deseos de las estudiantes.

En cuanto al rol de la escuela, sostengo que la institución ocupa un rol ambiguo. Por un lado, la escuela intenta acompañar esas maternidades y contenerlas dentro del espacio escolar, a través de un seguimiento individualizado de cada estudiante, lo

12 Aquí, con "nada", Ricardo se refiere a la casi nula asistencia de la estudiante. Más adelante, en la entrevista, me comentará que esa alumna, que resultó ser Sabrina, la estudiante mencionada anteriormente, asiste sólo una o dos veces por mes a clases. 
cual implica llamar a la estudiante, escuchar lo que necesita, contactar a la familia en caso de ser necesario, ir a su hogar, preparar actividades prácticas con la finalidad de levantar notas, etc. Por otro lado, la institución ve un problema a solucionar en estas experiencias de maternidad, ya que, según la escuela, tornan más dificultosa la continuidad de los estudios.

Ricardo: [Sabrina] "Vino un día por la libreta13, y yo no le puedo firmar una libreta por más que la aprecie, si no tengo una regularidad. Una regularidad son aproximadamente 20 días de asistencia en registro que no los tuve. Un día y nada, una vez al mes. Entonces yo no le puedo dar regularidad. Por ejemplo, me mandó al hermano ayer, que me mandó la libreta para que se la firme, y se la negué otra vez porque la verdad que es el único recurso que yo tengo para que un día venga"

Giuliana: "¿Y por qué considerás que hay chicas que son mamás y luego se les dificulta seguir viniendo?"

Ricardo: "Porque les interrumpe la función de madre de 24 horas del día".

En este extracto de entrevista, Ricardo continúa hablando del caso que menciona más arriba, el de Sabrina. El director sostiene que la firma de la libreta (firma que funciona como constancia de la regularidad de une alumne) es el único recurso que la escuela posee para asegurar la mínima asistencia de una alumna-madre. Sin embargo, Ricardo es plenamente consciente de que la maternidad no interrumpe la asistencia escolar de las jóvenes, sino que la escuela interrumpe las tareas maternas. Si bien las chicas desean continuar sus estudios y terminar el secundario, también desean quedarse en sus hogares con sus bebés lo cual, de alguna manera, refuerza el vínculo materno filial. En este marco, tiene sentido que Sabrina intente continuar

\footnotetext{
${ }^{13}$ Haciendo referencia a la Libreta de Asignación Universal por Hijo (AUH), asignación que cobra la mamá de Sabrina por ella y sus tres hermanes. La AUH para protección social es un seguro social de Argentina que otorga a personas desocupadas, con trabajo no registrado o que ganan menos del salario mínimo, vital y móvil un beneficio por cada hije menor de 18 años e hije con discapacidad. Entró en vigor el 29 de octubre de 2009 por el decreto 1602/09 del Poder Ejecutivo de la Nación. La Libreta -de cada une de les hijes- se presenta una vez por año y es obligatoria para cobrar el $20 \%$ que se acumula cada mes y continuar cobrando la Asignación al año siguiente. La Libreta posee tres partes referidas a: la situación laboral de la persona adulta responsable que cobra la Asignación; educación, para acreditar la asistencia de les niñes/adolescentes a la escuela; y salud, para certificar los controles y vacunaciones correspondientes
} 
sus estudios a distancia, yendo a cursar lo menos posible. Es en esta instancia en donde se produce un tira y afloje entre la escuela y las jóvenes. Si bien el director de la institución comprende las circunstancias en las que Sabrina se encuentra, también sabe que no puede mantener la regularidad de la estudiante con una asistencia mensual, por lo cual la negación a la firma de libretas es un recurso que Ricardo encuentra para "reencauzar" la situación y lograr que Sabrina asista a clases $^{14}$.

A diferencia de lo que sostienen muchas docentes, Ricardo no considera que las chicas que son madres jóvenes queden embarazadas por un desconocimiento o por irresponsabilidad. Él sostiene que "son decisiones" que se encuentran ligadas a una reproducción generacional de maternidades "jóvenes"15. En este sentido, sostengo que, si bien la escuela no pasiviza ni minimiza los deseos de las chicas al sostener (al menos dese la dirección institucional) que las maternidades en la adolescencia son decisiones, existen ciertos matices que colocan a la institución en un rol ambiguo en donde intenta acompañar esas decisiones al mismo tiempo que entra en tensión ellas.

\section{Conclusiones}

El objetivo de este texto ha sido analizar los modos en que las experiencias de maternidad y maternaje de un grupo de adolescentes escolarizadas de un barrio popular de Buenos Aires entraban en tensión con las distintas perspectivas que poseía el cuerpo docente en relación al embarazo en la adolescencia, así como también en qué medida estas experiencias de maternidad constituían (o no) un problema a solucionar. Tras el análisis se concluye que el embarazo en la adolescencia constituye, efectivamente, un problema en la medida en que pone en riesgo la continuidad de las chicas en la escuela. Sin embargo, como todo espacio social, la

\footnotetext{
${ }^{14}$ Al finalizar el año lectivo, durante el verano, hablé con Ricardo y charlamos sobre Sabrina y su situación pedagógica. Al preguntarle por lo que había sucedido finalmente con la libreta me comentó: "obviamente a fin de año se la firmé porque sé que ella y la familia lo necesitan".

${ }^{15}$ Sin embargo, en su gran mayoría, las madres de estas alumnas-madres intentan evitar que sus hijas queden embarazadas durante la adolescencia $y$, en muchos casos, se producen fuertes pleitos familiares cuando una joven anuncia inicialmente su embarazo.
} 
escuela se materializa como un espacio heterogéneo en donde se ponen en práctica diversas estrategias para mantener a las jóvenes en el aula. Mientras algunas docentes intentan evitar los embarazos considerados no deseados, interviniendo en las relaciones afectivas o aconsejando no tener relaciones sexuales casuales fuera de una relación de noviazgo estable; otres, como el director, despliegan estrategias, como el amague de no firmar la libreta de asistencias, con la finalidad de lograr que las alumnas-madres vuelvan a clases y cursen de forma regular.

De todas maneras, a pesar de que se desplieguen estrategias diversas y en momentos distintos (previo y posterior al embarazo), existe un criterio aunado sobre la importancia de intentar acompañar esas maternidades. En este sentido, propongo analizar las prácticas de prevención del embarazo, de control de la sexualidad de las jóvenes, y los intentos por contener a las chicas dentro del aula, no sólo como prácticas disciplinadoras, sino también como prácticas de cuidado. Resulta fundamental hacer esta lectura teniendo en cuenta las lógicas gerenciales que dejan a sujetes $y$, también en este caso, a instituciones teniendo que desarrollar estrategias para mantener a flote a las comunidades. En este marco, la escuela y sus docentes se ven con la necesidad de desarrollar determinadas prácticas con la finalidad de lograr (a veces sin mucho éxito) que sus estudiantes no queden embarazadas y que las alumnas-madres terminen sus estudios.

Las estudiantes, por otra parte, también desarrollan ciertas estrategias que desafían la mirada adulta, corriéndose del lugar de adolescentes que la escuela les asigna para reposicionarse en el de alumnas-madres, en donde se ensamblan diversos elementos identitarios tales como estudiante secundaria, adolescente y madre. En este sentido, resulta indispensable descartar las miradas condescendientes e infantilizantes que minimizan las perspectivas y decisiones de las jóvenes, y que catalogan de forma automática los embarazos en la adolescencia como tempranos, precoces o no deseados. Es necesario, por lo tanto, pensar la diferencia y comprender la feminidad, la adolescencia y/o la maternidad en la adolescencia en su amplia diversidad. Las asistencias intermitentes no implican necesariamente un desinterés por el estudio, sino que las dinámicas de la escuela, y sus requerimientos de asistencia para lograr obtener una regularidad, interrumpen el trabajo materno, generando modos particulares de habitar la escuela siendo madre adolescente que deben ser tenidos en cuenta. Esto es, para que docentes y directives no deban llevar a cabo 
malabarismos cotidianos (Grinberg, 2011) sería necesario desarrollar condiciones y políticas públicas específicas que permitieran a la escuela realizar estos acompañamientos.

\section{Bibliografía}

Adaszko, A. (2005). Perspectivas socio-antropológicas sobre la adolescencia, la juventud y el embarazo. En: Gogna, M. (comp.) Embarazo y maternidad en la adolescencia. Estereotipos, evidencias y propuestas para políticas públicas, pp. 33-66. Buenos Aires: CEDES.

Barrett, G. \& Wellings, K. (2002). What is a "planned" pregnancy? Empirical data from a British study. En: Social Science \& Medicine 55, pp. 545-557.

Bourdieu, P. (2001) [1985]. Lenguaje y poder simbólico. En: ¿Qué significa hablar? Economía de los intercambios lingüísticos, pp. 63-104. Ediciones Akal. Madrid.

Casas Alvarez, J. \& Solorio Perez, C. (2015). Jóvenes en edad escolar y embarazo no $\begin{array}{lllll}\text { planeado. } & \text { Reflexiones, } & \text { 79-87. }\end{array}$ https://doi.org/10.15517/rr.v94i2.25457

Cono Sur (2017). Prevención y reducción del embarazo no intencional en la población adolescente del Cono Sur. Un marco estratégico subregional para mejorar la implementación de la política local. UNFPA. Asunción, Junio de 2017.

Crenshaw, K. (1989). Demarginalizing the intersection of race and sex: A black feminist critique of antidiscrimination doctrine, feminist theory and antiracist politics. University of Chicago Legal Forum. 1989(1), pp.139-167.

Czerniuk, R. (2016). Maternidad adolescente e identidad en contextos de pobreza. Anuario de Investigaciones, XXIV, pp. 79-88.

Dafunchio, S. \& Grinberg, S. (2013). Biopolítica y experiencia de la escolaridad en contextos de extrema pobreza urbana y degradación ambiental. Magistro, 7(14), pp. 245-269.

Fainsod, P. (2008). Embarazos y maternidades adolescentes. Desafíos de las escuelas. En: Morgade, G. \& Alonso, G. (comp.) Cuerpos y sexualidades en la escuela. De la "normalidad" a la disidencia. Editorial Paidós. Buenos Aires. 
Extraído

de:

http://www.edumargen.org/docs/2018/curso25/unid04/apunte05_04.pdf

Furstenberg, F. (2003). Teenage childbearing as a public issue and private concern Annual Review of Sociology, 29, pp. 23-29. https://doi.org/10.1146/annurev.soc.29.010202.100205

García Hernández, G. (2014). Embarazo adolescente y pobreza. Una relación compleja. Iztapalapa Revista de Ciencias Sociales y Humanidades, 77 , pp. 1353. https://doi.org/10.28928/ri/772014/atc1/garciahernandeze

Geronimus, A. (2004). Teenage childbearing as cultural prism. British Medical Bulletin, 69, pp. 155-166. https://doi.org/10.1093/bmb/ldh019

Grinberg, S. (2009). Políticas y territorios de escolarización en contextos de extrema pobreza urbana: Dispositivos pedagógicos ente el gerenciamiento y la abyección. [En línea] Archivos de Ciencias de la Educación (4a. época), 3(3). Extraído de: http://www.fuentesmemoria.fahce.unlp.edu.ar/art_revistas/pr.4084/pr.4084.p df

Grinberg, S. (2011). Gubernamentalidad y educación en tiempos de gerenciamiento. Reflexiones en torno de la experiencia de los dispositivos pedagógicos en contextos de extrema pobreza urbana. Presentado en: Departamento de Ciencias de la Educación. Facultad de Humanidades y Ciencias de la Educación Universidad Nacional de La Plata. VIII Encuentro de Cátedras de Pedagogía de Universidades Nacionales Argentinas. Teoría, formación e intervención en Pedagogía. La Plata, 8, 9 y 10 de agosto de 2011. Extraído de: http://ecpuna.fahce.unlp.edu.ar. ISSN 18539602.

González Espinosa, M. (2020). Lo que viene después del embarazo adolescente: Un análisis de la situación de las adolescentes madres en el Perú y un modelo de éxito en Argentina. Revista Solonik. Políticas públicas y derechos humanos, 7, pp. 70-85.

Langer, A. (2002). El embarazo no deseado: impacto sobre la salud y la sociedad en América Latina y el Caribe. Rev Panam Salud Publica/Pan Am J Public Health 11(3), pp.192-205. https://doi.org/10.1590/s1020-49892002000300013 
Llanes Díaz, N. (2012). Ser madre adolescente y dejar la escuela: Replanteamiento y consideraciones. Presentado en: V Congreso de la Asociación Latinoamericana de Población, Montevideo, Uruguay, 23 al 26 de octubre de 2012.

Mariño, M. (2013). El embarazo precoz: no querido pero deseado. Universitas,

Revista de ciencias sociales y humanas, 18, pp. 103-131. https://doi.org/10.17163/uni.n18.2013.04

Muñoz, A. (2017). La sociedad de control: una mirada a la educación del siglo XXI desde Foucault. Rev. filos., 73. Santiago oct. 2017. http://dx.doi.org/10.4067/S0718-43602017000100317.

Nóblega, M. (2009). La maternidad en la vida de las adolescentes: implicancias para la acción. Revista de Psicología, XXVII (1), pp. 30-54, Pontificia Universidad Católica del Perú. Lima, Perú. https://doi.org/10.18800/psico.200901.002

Ojeda Álvarez, D. \& Cruz Moya, O. (2004). "Yo me parto": oralidad, humor, gramática y pragmática, un coctel lúdico para el aula de e/le. Actas del XV Congreso Internacional de Ásele.

Oviedo, M. \& García, M. (2011). El embarazo en situación de adolescencia: una impostura en la subjetividad femenina. Revista Latinoamericana de Ciencias Sociales, Niñez y Juventud, 9(2) pp. 929-943, Centro de Estudios Avanzados en Niñez y Juventud. Manizales, Colombia.

Pantelides, E. (2004). Aspectos sociales del embarazo y la fecundidad adolescente en América Latina. Notas de Población, Naciones Unidas Comisión Económica para América Latina y el Caribe (CEPAL), pp. 7-33.

Pantelides, E. y Binstock, G. (2007). La fecundidad adolescente en la Argentina a comienzos del siglo XXI. Revista Argentina de Sociología 9, pp. 24-43.

Parra Abaúnza, N. (2012). Cuando el embarazo no planificado se desea. Estudio aproximativo sobre la vivencia de adolescentes embarazadas. Documentos de Trabajo Social, 51, pp. 181-203.

Posada, C. (2014). Embarazo en la adolescencia: no una opción, una falta de opciones. Revista sexología y sociedad, 10 (25), pp. 4-10.

Rondón, A. \& Betancur, H. (2015). El embarazo a temprana edad, un análisis desde 
la perspectiva de madres adolescentes. Revista virtual Universidad Católica del Norte, 44, pp. 222-237.

Scharagrodsky, P. (2005). Cuerpo, género y poder en la escuela. El caso de la educación física escolar argentina (1880-1930). Ethos educativo 33/34, pp. 3967. https://doi.org/10.15448/1980-864x.2001.2.24437

Stern, C. \& García, E. (2001). Hacia un nuevo enfoque en el campo del embarazo adolescente. En: C. Stern \& J. Figueroa (Coords.) Sexualidad y salud reproductiva. Avances y retos para la investigación, (pp. 331-358). México, D. F.: El Colegio de México.

Unicef (2019). Estadísticas de los hechos vitales de la población adolescente en la Argentina. Fondo de las Naciones Unidas para la Infancia (UNICEF). Julio de 2019.

Vélez E. \& Figueredo L. (2015). Transmisión generacional de la pobreza y vulnerabilidad en las adolescentes embarazadas. Revista Educación en Valores. Universidad de Carabobo, 2(24), pp. 50-57.

Weisbrot, M. A., Fraguasa, L., Espósitoa, M. E., Camoiranoa, J., Sciarretaa, S., Graneroa, M., \& Weisbrota, M. V. (2019). Percepciones de los adolescentes del Bajo Boulogne, Buenos Aires, Argentina, sobre el embarazo en la adolescencia, estudio cualitativo. Arch Argent Pediatr 117(5), pp. 314-322.

Zamberlín, N. (2005). Percepciones y conductas de las/los adolescentes frente al embarazo y la maternidad/paternidad. En: Gogna, M. (comp.) Embarazo y maternidad en la adolescencia. Estereotipos, evidencias y propuestas para políticas públicas, pp. 285-316. Buenos Aires: CEDES. 